\title{
Predicting Product Distribution of Propene Dimerization in Nanoporous Materials
}

\author{
Yifei Michelle Liu ${ }^{\dagger}$ and Berend Smit*, ${ }^{\dagger},+\circ$ \\ ${ }^{\dagger}$ Department of Chemical and Biomolecular Engineering, University of California, Berkeley, California 94720, United States \\ ${ }^{\ddagger}$ Laboratory of Molecular Simulation, Institut des Sciences et Ingénierie Chimiques, École Polytechnique Fédérale de Lausanne \\ (EPFL), Rue de l'Industrie 17, CH-1951 Sion, Switzerland
}

\section{Supporting Information}

ABSTRACT: In this work, a theoretical framework is developed to explain and predict changes in the product distribution of the propene dimerization reaction, which yields a mixture of $\mathrm{C}_{6}$ olefin isomers, resulting from the use of different porous materials as catalysts. The MOF-74 class of materials has shown promise in catalyzing the dimerization of propene with high selectivity for valuable linear olefin products. We show that experimentally observed changes in the product distribution can be explained in terms of the contribution of the pores to the free energy of formation, which are directly computed using molecular simulation. Our model is used to screen a library of 118 existing and hypothetical MOF and zeolite structures to study how product
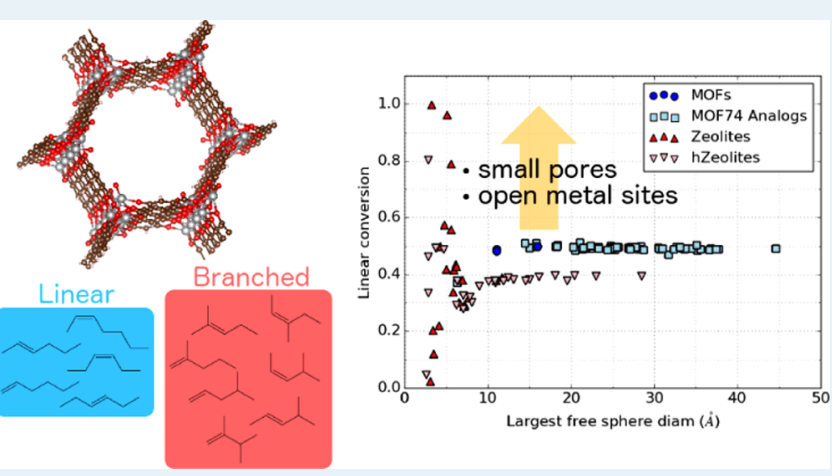
distribution can be tuned by changing pore size, shape, and composition of porous materials. Using these molecular descriptors, catalyst properties are identified that increase the selective reaction of linear olefin isomers, which are valued as industrial feedstocks. A pore size commensurate with the size of the desired linear products enhances linear conversion by sterically hindering the branched isomers. Another promising feature is the presence of open metal sites, which interact with the olefin $\pi$ bond to provide favorable binding sites for the linear isomers.

KEYWORDS: propene dimerization, metal-organic frameworks, zeolites, product distribution, Monte Carlo, molecular simulation

\section{INTRODUCTION}

Metal-organic frameworks (MOFs) are porous, crystalline materials comprising a three-dimensional (3D) network of metal centers (nodes) connected by organic linkers. MOFs have generated a great deal of interest as a promising class of materials for gas separation and storage applications, because of their high surface area for gas adsorption and the virtually limitless design space of nodes and linkers. ${ }^{1}$ The same characteristics make MOFs attractive for catalysis as well, and recent years have seen the synthesis of frameworks, which have high chemical and thermal stability and accessible metal sites that can serve as active sites for catalysis. ${ }^{2-5}$

For example, the MOF-74 class of materials, which features coordinatively unsaturated metal sites, has shown promise in catalyzing the dimerization of propene to $\mathrm{C}_{6}$ olefins with relatively high selectivity and activity for valuable linear products: 1-hexene is a feedstock for polyethylene, and its linear regioisomers are used to produce lubricants and detergents. ${ }^{6}$ Supported nickel catalysts are typically preferred for propene dimerization and other olefin oligomerization reactions, ${ }^{6-8}$ but there is often a tradeoff between catalytic activity and selectivity for the desired products: Mlinar et al. showed, via tuning the free volume of a $\mathrm{Ni}-\mathrm{Na}-\mathrm{X}$ zeolite, that the most constricted pores had the highest fraction of linear isomer products, but the lowest catalytic activity. Correspondingly, increasing the pore space led to higher activity but a loss of selectivity toward linear isomers. ${ }^{9}$ Subsequent work has shown that $\mathrm{Ni}_{2}$ (dobdc) (Ni-MOF-74) and its expanded-linker analogue, $\mathrm{Ni}_{2}$ (dobpdc) (Ni-MOF-274), can achieve high linear selectivity while maintaining high catalytic activity. ${ }^{10}$ By analyzing the product distribution of propene oligomerization in the MOFs, Ni-Na-X, and Ni-loaded MCM-31, Mlinar et al. showed that both MOF-74 materials had $\sim 10 \%$ less dimer branching than the zeolite and mesoporous materials. The lower dimer branching and corresponding higher linear selectivity in MOFs was hypothesized to be due to increased steric hindrance around the $\mathrm{Ni}^{2+}$ active sites in the MOFs, which favors the transition state for the linear dimer. ${ }^{10}$

The phenomena governing reaction product distributions encompass a wide range of time and length scales, from the localized and short-lived transition state, to adsorption inside an individual pore and product diffusion out of the porous material. An important difference of reactions in nanopores is that, unlike reactions in liquid or gas phase, the thermody-

Received: March 5, 2017

Revised: April 21, 2017

Published: April 28, 2017 
namics of the reaction is not known. In particular, there is a lack of information on the contribution of the MOF or zeolite to the free energy of formation. Molecular simulations can compensate for this missing information and can help to, for example, quantify the extent to which the pores favor or disfavor the products that can form. Insights gained from molecular simulations can also provide an atomistic perspective on features such as pore shape and composition in order to better understand how these descriptors influence product distribution and possibly even predict higher-performing candidate materials for propene dimerization and other oligomerization reactions. ${ }^{11,12}$

Expanding on the foundational ideas of shape selectivity, in this work, we show how the product distribution can be tuned as a function of pore size, shape, and composition. We used molecular simulations to compute the contribution of different porous materials to the free energy of formation of the $\mathrm{C}_{6}$ olefin isomers. We consider the MOF Ni-MOF-74 and the zeolite Ni$\mathrm{Na}-\mathrm{X}$ in the Mlinar study, ${ }^{10}$ as well as synthesized and hypothetical zeolites and hypothetical MOF-74 analogues, for a total of 118 materials. Where experimental results are available, we show that our computational predictions for linear selectivity agree well with the experiment. One of the questions we sought to address, for example, is how the linker structure in $\mathrm{Ni}-\mathrm{MOF}-74$ influences the free energy of formation, prompting us to study the MOF-74 analogue materials generated by Witman et al. ${ }^{13}$ Similar considerations motivated our choice of additional existing and hypothetical zeolite materials. ${ }^{14,15}$ Of particular importance in this work is the presence of coordinatively unsaturated metal sites in the MOF-74 analogues. These "open" metal sites can engage in $\pi$ complexation with the double bond of the olefins, causing enrichment of olefin adsorption. ${ }^{16}$ We demonstrate that the presence of open metal sites complements the shape-selective effects of pore geometry in determining reaction product distribution.

\section{METHODS}

2.1. Adsorbate and Adsorbent Models. To compute the free energy of formation of the different isomers in each porous material, we used models that describe the equilibrium interactions between the adsorbates and the adsorbent materials. Parameters for both bonded and van der Waals interactions for the dimer isomers were taken from the TraPPE united atom force field, which has been demonstrated to reproduce experimental hydrocarbon isotherms in porous materials well. ${ }^{17}$

Nonbonded parameters for Ni-MOF-74 and Ni-MOF-274 were taken from the Dreiding force field, ${ }^{18}$ with the exception of the metal atoms for which parameters were taken from UFF. ${ }^{19-22}$ Charges for these MOFs were calculated using the REPEAT method ${ }^{23}$ with electrostatic potentials computed using density functional theory (DFT). ${ }^{24}$ MOF-74 analogue nonbonded parameters and charges were calculated by Witman et al. $^{13}$

Zeolite-adsorbate interactions were described using modified alkene-zeolite parameters in Liu et al. ${ }^{25}$ In order to capture the preferential interaction between the MOF open metal sites and the olefin double bonds, the double bond was described by a three-point charge model $\left(q_{\mathrm{CH} x}=0.85 e\right.$ and $\left.q_{\mathrm{COM}}=-1.70 \mathrm{e}\right),{ }^{26}$ which has been shown to reproduce the experimental adsorption isotherm for ethene in the open-metal site $\mathrm{MOF} \mathrm{Cu}_{3}(\mathrm{BTC})_{2}{ }^{27}$ For all other guest-adsorbate interactions, Lorentz-Berthelot mixing rules were used to compute Lennard-Jones interaction parameters.

2.2. Computing Product Distribution. We consider the product distribution to comprise the 12 experimentally detected isomers shown in Table $1 .^{10}$ Therefore, we do not

Table 1. Propene Dimer Isomers Considered in This Work

$\begin{array}{cc}\text { Isomer } & \text { Abbreviation } \\ \text { 1-hexene } & 1 \mathrm{~h} \\ \text { cis-2-hexene } & \mathrm{t} 2 \mathrm{~h} \\ \text { trans-3-hexene } & \mathrm{c} 2 \mathrm{~h} \\ \text { cis-3-hexene } & \mathrm{t} 3 \mathrm{~h} \\ \text { 2-methyl-2-pentene } & \mathrm{c} 3 \mathrm{~h} \\ \text { 4-meth-methyl-2-pentene } & \mathrm{c} 3 \mathrm{~m} 2 \mathrm{p} \\ \text { 2-methyl-1-pentene } & 4 \mathrm{~m} 1 \mathrm{p} \\ \text { cis-4-methyl-2-pentene } & \mathrm{c} 4 \mathrm{~m} 2 \mathrm{p} \\ \text { trans-4-methyl-2-pentene } & \mathrm{t} 4 \mathrm{~m} 2 \mathrm{p}\end{array}$

consider isomers that might have a very low free energy, but are not formed in the experimental reaction pathway, or that do not diffuse out of the material. We are interested in predicting the fraction of linear isomers $x_{l}$ in the product distribution arising from the different porous materials. The first step in any study of product distribution is normally the free energy of formation. Since this free energy is not known experimentally, we use molecular simulations to gain insight into how the pores influence the product distribution by considering the pore's contribution to the free energy of formation. Each product isomer, once formed, must spend time adsorbed inside the material before diffusing out. Therefore, the free energy of formation inside the pore can be expressed for product isomer $i$ as

$$
\Delta G_{\text {form }, i}=\Delta G_{\text {form }, i}^{\mathrm{gas}}+\Delta G_{\mathrm{ads}, i}-\Delta G_{\mathrm{ads}, \text { reac }}
$$

Note that, for all product isomers, $\Delta G_{\text {ads,reac }}$ is the same, since it refers to adsorption of two reactant propene molecules, so the dominant influence of the pore on the product distribution is captured by the free energies of adsorption of the product isomers, $\Delta G_{\text {ads }, i}$.

At this point, it is important to note that thermodynamics is not the only factor that determines product distribution. Kinetics can also play a role, but even in a kinetically controlled 
reaction, if the transition state resembles the product state, then the contribution of the pores to the free energy of formation can be of use, i.e., assuming that, for this system, the BrønstedEvans-Polanyi relation holds. ${ }^{28}$ Without knowing the precise mechanism of reaction, we can quantify the probability of formation of a particular isomer $i$ as

$$
p_{i} \propto \exp \left(-\beta \Delta G_{\mathrm{ads}, i}\right)
$$

by assuming that the reaction is rate-limited, which is likely given the small reactor size, ${ }^{10}$ and that the transition state resembles the product molecule.

The standard free energy of adsorption $\left(\Delta G_{\mathrm{ads}}\right)$ was calculated as

$$
\Delta G_{\mathrm{ads}}=G_{\mathrm{ex}}-G_{\mathrm{ex}}^{\mathrm{gas}}
$$

where excess free energies of the dimer isomers at infinite dilution inside the frameworks were computed via Widom insertions to get the Henry coefficient, ${ }^{29}$

$$
k_{\mathrm{H}}=\beta \frac{\left\langle W_{\mathrm{H}}\right\rangle}{\left\langle W_{\mathrm{IG}}\right\rangle}
$$

where $\left\langle W_{\mathrm{IG}}\right\rangle$ is the ideal-gas Widom Rosenbluth weight, computed via a separate simulation in an empty box. From the Henry coefficient, the excess chemical potential $\left(\mu_{\mathrm{ex}}\right)$ was calculated using

$$
k_{\mathrm{H}}=\beta \exp \left(-\beta \mu_{\mathrm{ex}}\right)
$$

For a single molecule at infinite dilution, $\mu_{\mathrm{ex}}=G_{\mathrm{ex}} \cdot \mu_{\mathrm{ex}}^{\mathrm{gas}}=G_{\mathrm{ex}}^{\mathrm{gas}}$ was also computed via Widom insertions in the gas phase.

Internal energies of adsorption were computed by using the Boltzmann-weighted average energy from Widom insertion, $\langle U\rangle_{\mathrm{W}}$, and then subtracting the ideal gas intramolecular energy calculated from NVT simulation to obtain

$$
\Delta U_{\mathrm{ads}}=\langle U\rangle_{\mathrm{W}}-\left\langle U_{\mathrm{IG}}\right\rangle
$$

From these values, the enthalpy and entropy of adsorption could be calculated using relations from classical thermodynamics:

$$
\Delta H=\Delta U-k_{\mathrm{B}} T
$$

and

$$
\Delta G=\Delta H-T \Delta S
$$

where eq 7 applies for adsorption at zero coverage. ${ }^{30,31}$

Configurational-bias Monte Carlo (CBMC) simulations ${ }^{29}$ were conducted with the RASPA molecular simulation package. $^{32}$ Simulations were conducted at $450 \mathrm{~K}$, the operating temperature of the propene dimerization reaction. At least 1000000 Monte Carlo (MC) cycles were used to ensure convergence of the Henry coefficients. Adsorption sites were visualized using the VisIt package. ${ }^{33}$

\section{RESULTS AND DISCUSSION}

3.1. Structure Database. Since our simulations are at equilibrium conditions, we chose chemical structures that would be representative of the equilibrium environment felt by the product olefin isomers. The 118 total porous materials considered in this study are summarized in Table 2 and Figure 1. ${ }^{14,15,34,35}$ Zeolite structures are represented in our simulations without $\mathrm{Ni}$ sites, as the loading of $\mathrm{Ni}$ in $\mathrm{Ni}-\mathrm{Na}-\mathrm{X}$ is $<0.6 \mathrm{wt} \%$, compared with 37.7 wt $\%$ in Ni-MOF-74. ${ }^{10}$ In addition, with the exception of Ni-MOF-74, MOFs containing Mg rather than
Table 2. Materials Screened

\begin{tabular}{lc}
\multicolumn{1}{c}{ material class } & count \\
MOF-74 & $3^{a}$ \\
hypothetical MOF-74 analogues & $63^{b}$ \\
zeolites & $23^{c}$ \\
hypothetical zeolites & $29^{d}$
\end{tabular}

${ }^{a}$ M-MOF-74 [M = Ni, Mg], ${ }^{34}$ Mg-MOF-274. ${ }^{35}{ }^{b}$ MOF-74 analogues assembled by Witman et al. ${ }^{13,36}{ }^{c^{S}}$ Selected from IZA database with a range of pore sizes. ${ }^{14} d$ All $1 \mathrm{D}$ channel topology. ${ }^{15}$
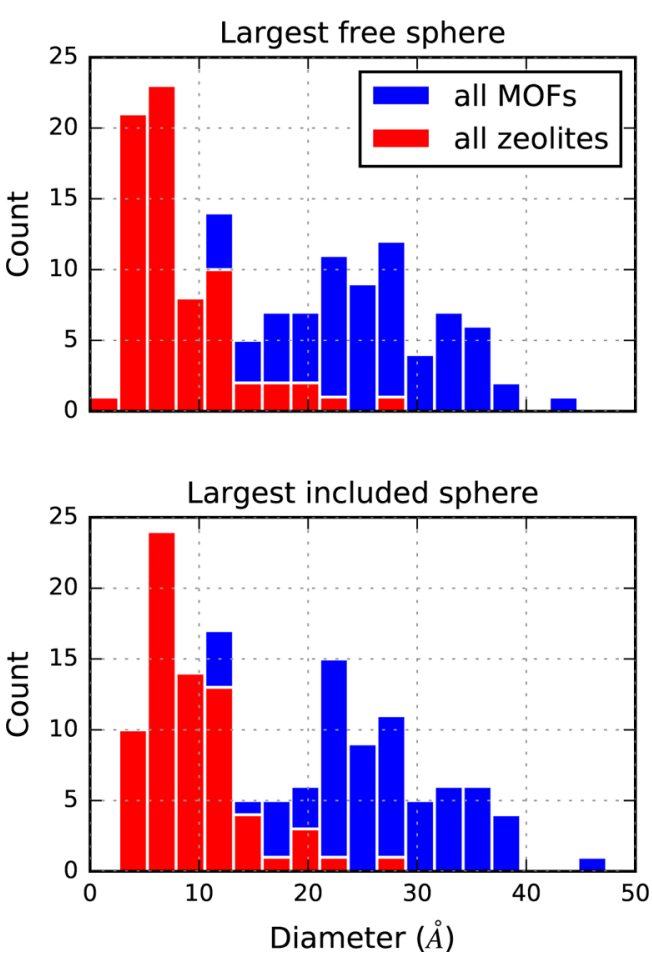

Figure 1. Geometric pore descriptors summary. Channel diameter is gauged by the largest free sphere diameter $\left(d_{\text {free }}\right)$, which is the size of the largest probe sphere able to freely percolate through the material.

$\mathrm{Ni}$ as the metal site are used, since structures and parameters have been generated for a large set of Mg-based MOF-74 isostructural analogues, ${ }^{13}$ and we find that changing the metal has little effect on the equilibrium properties of the material (Figure 2).

3.2. Free-Energy Profiles. The striking observation that motivated this work is the enhanced linear conversion of $\mathrm{Ni}$ MOF-74 and Ni-MOF-274, compared to Ni-Na-X in the work of Mlinar et al. ${ }^{10}$ They showed via fixed-bed reactor experiments that Ni-MOF-74 and Ni-MOF-274 had a lower degree of dimer branching than the zeolite $\mathrm{Ni}-\mathrm{Na}-\mathrm{X}$, while $\mathrm{Ni}$ MOF-74 and Ni-MOF-274 had a similar degree of dimer branching of $\sim 38 \% .^{10}$ In a homogeneous reaction, one would begin a study of the product distribution by comparing the free energies of formation of the products. For a reaction in a porous material, however, this thermodynamic information is not known. The product distribution may be influenced by many mechanisms, including thermodynamics, kinetics, and diffusive processes. However, if the enhancement of linear conversion in the MOFs is governed by the free energy of formation, then we should be able to see a more favorable free energy of linear isomers in the MOFs reflected in our calculations. 


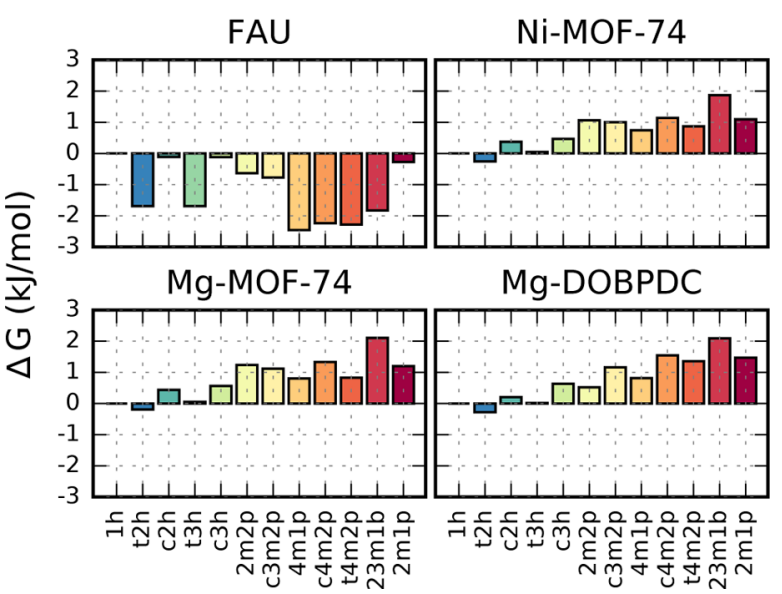

Figure 2. Free-energy profile of adsorbates in porous frameworks, relative to 1 -hexene.

The $\Delta G_{\text {ads }}$ computed for the five linear and seven branched olefin isomers (Table 1) are shown in Figure 2 for Ni-MOF-74, Mg-MOF-74, Mg-MOF-274, and FAU zeolite, relative to the $\Delta G_{\text {ads }}$ of 1-hexene. This "free-energy profile" represents the degree to which the porous material stabilizes the formation of each isomer. An important result is that this figure shows the free-energy profiles are qualitatively different between the three MOF structures and FAU zeolite. In the MOFs, the linear isomers are, on the whole, more stabilized than the branched isomers, with the exception of cis-3-hexene in Mg-MOF-274. The free-energy profile of the MOFs is virtually the inverse of that of FAU, where, with the exception of trans-2-hexene and trans-3-hexene, the linear isomers have much higher $\Delta G_{\text {ads }}$ values than the branched isomers. The free-energy profiles MOFs in Figure 2 look similar to each other; indeed, the rootmean-square error (RMSE) between the Ni-MOF-74 and MgMOF-74 (Mg-MOF-274) is only $0.12(0.28) \mathrm{kJ} / \mathrm{mol}$, compared to $2.22 \mathrm{~kJ} / \mathrm{mol}$ between Ni-MOF-74 and FAU, indicating that the identity of the metal does not influence the equilibrium free energy of the isomers. Overall, these findings are consistent with the results of the experimental study of Mlinar et al., ${ }^{10}$ giving us confidence that our approach is reasonable and can be applied to other materials that may be promising for this reaction.

The source of the contrast in free-energy landscapes of the metal-organic and zeolitic materials is primarily in differences in the enthalpy of adsorption. Figure 3 shows $\Delta H_{\text {ads }}$ and $\Delta S_{\text {ads }}$ of FAU and Ni-MOF-74. The enthalpy profile of FAU looks qualitatively similar to its free-energy profile; branched products have, on average, more favorable enthalpies of adsorption, compared to linear products. The same qualitative agreement holds for Ni-MOF-74. However, for the entropic contribution, the qualitative trends are different. Adsorption of branched products is more entropically favored in Ni-MOF-74 and FAU does not display a clear entropic preference toward the adsorption of either group. The trends in entropy of adsorption can be attributed to the larger configurational space of the linear molecules in the gas phase, which means that, once adsorbed, they lose more entropy than their branched, more rigid, counterparts. Enthalpically, the flexibility of the linear isomers allows them to adopt an energetically favorable configuration with the $\pi$-bond closer to the open metal sites in the MOF, whereas FAU has no such favorable adsorption sites, so the linear isomers lose more entropy, relative to the

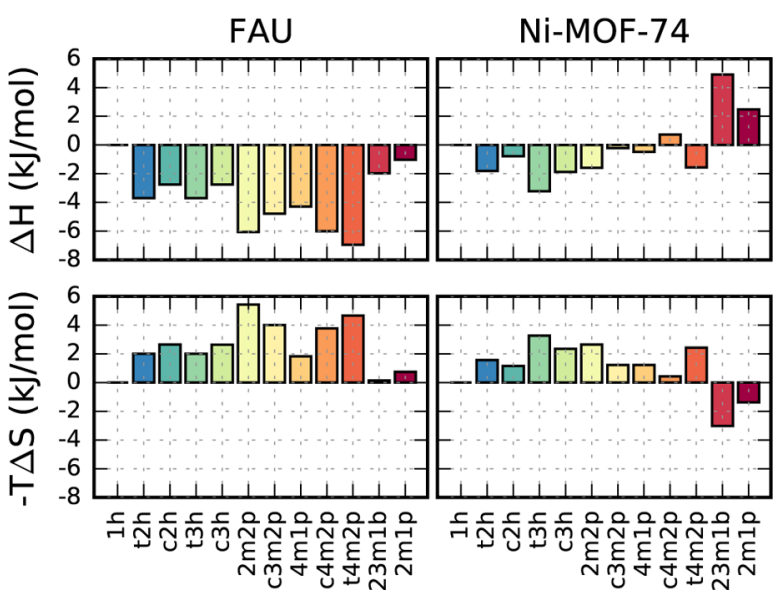

Figure 3. Enthalpic $(\Delta H)$ and entropic $(-T \Delta S)$ contributions to the free-energy profile, relative to 1 -hexene.

branched isomers upon adsorption in the MOFs compared to that observed in the zeolites.

3.3. Linear Conversion. We wish to identify materials that can selectively convert propene to linear $\mathrm{C}_{6}$ olefins. We quantify this selectivity by calculating the total fraction of linear isomers in the product mixture predicted by molecular simulation. Making use of eq 2 for the probability of formation of a particular isomer $p_{i}$, and defining the linear conversion as $x_{L}=\sum_{\text {linear }} p_{i}$, we find that

$$
x_{L}=\frac{\sum_{\text {linear }} \exp \left(-\beta \Delta G_{i, \text { ads }}\right)}{\sum_{\text {all }} \exp \left(-\beta \Delta G_{i, \text { ads }}\right)}
$$

Linear conversion $x_{L}$ calculated in this manner for Ni-MOF74, Mg-MOF-74, Mg-MOF-274, and FAU is shown in Figure 4,

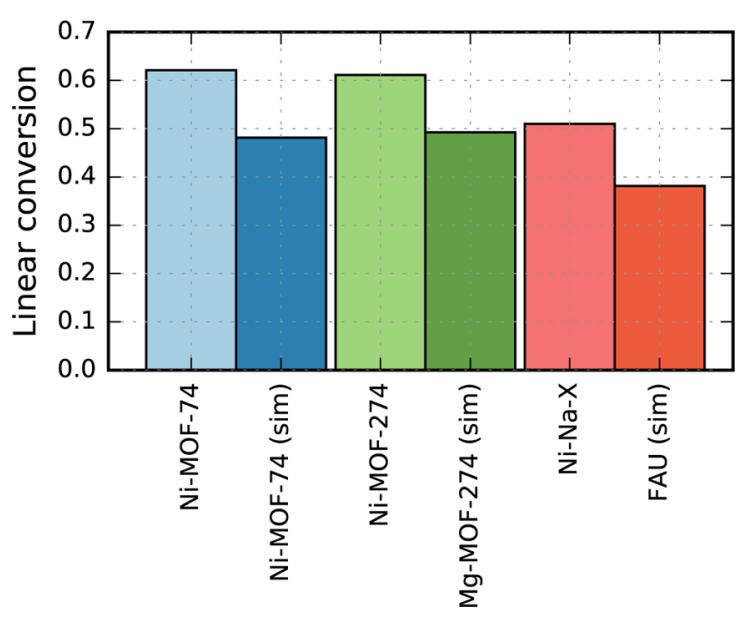

Figure 4. Fraction of linear dimer isomers in product distribution, experimental and simulated. Experimental values are taken from the work of Mlinar et al. ${ }^{10}$

together with the equivalent experimental values from Mlinar et al. ${ }^{10}$ The computed linear conversions for the MOF materials are close in value, while the linear conversion of FAU is $~ 10.0 \%$ lower. This agrees with the observation from the previously calculated free-energy profiles that the MOFs favor adsorption of linear isomers to a larger degree than branched isomers.

The $10.0 \%$ enhancement in linear conversion of Ni-MOF-74, relative to $\mathrm{FAU}$, is in good agreement with the $11.1 \%$ 
enhancement observed experimentally by Mlinar et al., ${ }^{10}$ suggesting that our model and theoretical framework capture quantitatively the influence of the adsorbent material on product distribution. Interestingly, the absolute predicted linear conversions are $13.0 \%$ lower than their respective experimental values. This means the experimental materials are consistently more selective toward linear isomers than their simulated counterparts. This could be due to diffusive barriers that are not taken into account by our simulations, which would favor the less-bulky linear isomers. We also want to emphasize that these calculations rely on equilibrium thermodynamics, and thus presume that Brønsted-Evans-Polanyi conditions are met. This is often, but not always, true when the product and transition states are similarly affected by steric hindrance; however, a rigorous validation of BEP relation applicability would require quantum mechanical study of each material. ${ }^{12}$ In the propene dimerization reaction, experimental results suggest that increased linear selectivity is linked to increased steric constraints within a material, which disfavors dimer branching. ${ }^{9}$ Since we observe that the steric penalty imposed on the product molecules is the dominant factor leading to trends in linear selectivity that agree with the experiment, we infer that it is reasonable to apply BEP assumptions to propene dimerization within the materials studied.

From this comparison with the experimental results of Mlinar et al., ${ }^{10}$ we can conclude that the contribution of the pores to the free energy of formation is indeed a useful indicator of how the product distribution varies with the catalyst material. Therefore, it is interesting to explore how this distribution can be further changed by using a different zeolite or MOF structure. Expanding the database of structures allows us to better understand how molecular descriptors correlate with product distribution, and further elucidate design principles of materials for propene dimerization.

3.4. Extension to Other Frameworks and Understanding Factors Influencing Linear Selectivity. A wide variety of pore geometries exists among crystalline, microporous materials. Differences in local structure around a particular adsorption site, as well as the global topology of the material, influence the relative adsorption free energies within a set of adsorbate molecules. Most of the frameworks in this study have one-dimensional channel topologies so that we could use the channel diameter as a quantitative metric for comparison between materials, and compare these materials to the MOF-74 class of materials, which have high experimentally observed linear selectivity.

The linear conversions computed for these materials in this study are shown in Figure 5, plotted against the largest free sphere diameter $\left(d_{\text {free }}\right)$. The zeolite materials, both hypothetical and synthesized, show sensitivity to $d_{\text {free }}$ below $10 \AA$, and above this threshold diameter, the linear conversion remains virtually constant. Below $d_{\text {free }}=10 \AA$, the linear conversion varies nonmonotonically with pore size. At intermediate diameters, the materials favor branched dimer isomers, and there is a local minimum in linear conversion at $\sim 7.5 \AA$. From $7.5 \AA$ to $5 \AA$, the linear conversion increases, reaching almost $80 \%$. Below 5 $\AA$, there is no clear correlation between linear conversion and pore size; instead, there is a wide spread in predicted product distributions, likely because these small pore diameters approach the average size of a $\mathrm{C}_{6}$ olefin isomer and small changes in local pore geometry can cause some $\mathrm{C}_{6}$ olefin isomers to be extremely disfavored.

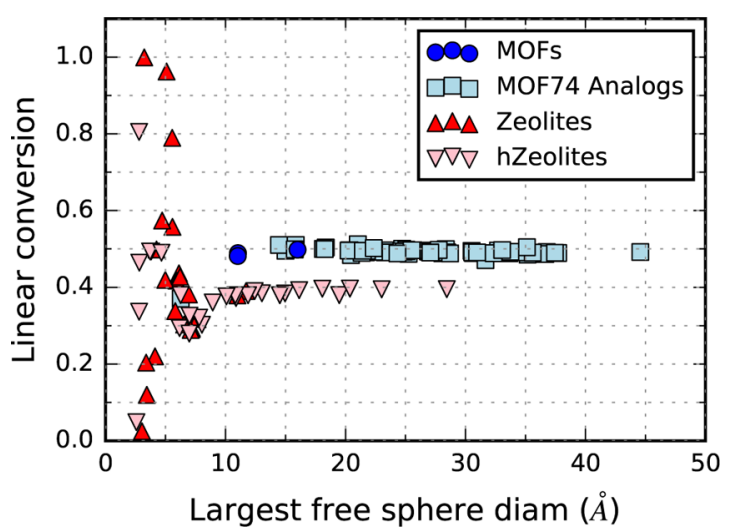

Figure 5. Fraction of linear dimer isomers in product distribution (linear conversion) versus largest free sphere diameter for zeolites and MOFs.

There are a few zeolites that achieve close to full linear conversion (APD, 99\%, and ATO, 96\%) and zero linear conversion (EDI, 2.5\%, and h8186492, 4.8\%). Their structures shown in Figure 6. The pores of APD and ATO zeolites

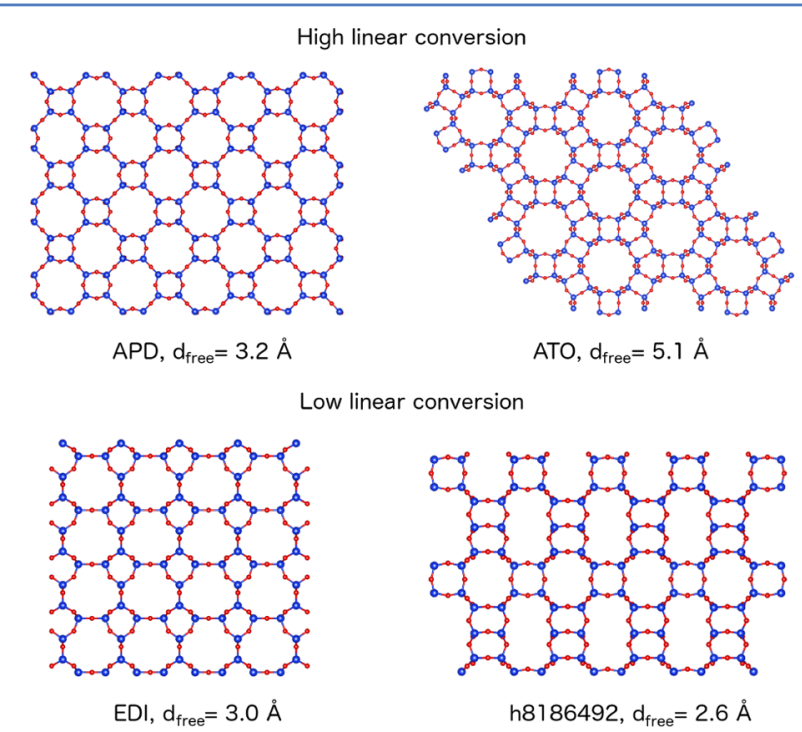

Figure 6. Zeolites achieving close to $100 \%$ and $0 \%$ linear conversion.

provide particularly favorable adsorption sites for 1-hexene, cis2-hexene, and cis-3-hexene, which are able to coil into the small channels without incurring too much steric penalty, in order to take advantage of the enthalpic reward of binding in a tight pocket. In APD (ATO), the $\Delta H_{\text {ads }}$ value of these three linear isomers is, on average, $19 \mathrm{~kJ} / \mathrm{mol}(5.5 \mathrm{~kJ} / \mathrm{mol})$ more favorable than the next-closest isomer's $\Delta H_{\text {ads }}$ value.

For EDI and h8186492 zeolites, the product distributions are heavily dominated by a single-branched isomer: 2,3-methyl-1butene in EDI and cis-4-methyl-2-pentene in h8186492, which respectively account for $87 \%$ and $88 \%$ of the total product distribution. These branched isomers are inherently more configurationally constrained, so we attribute their comparatively low $\Delta H_{\text {ads }}$ to a fortuitous binding pocket that complements the isomer structure. These examples demonstrate that frameworks whose pore sizes approach the size of the product molecules can be highly selective toward a particular product or group of products, with very different 
selectivities even among structures with comparable geometric descriptors.

Like the zeolites, Ni-MOF-74 and its isostructural analogues also have approximately constant linear conversion above $10 \AA ̊$. Surprisingly, however, the linear conversion is shifted $10 \%-$ $13 \%$ higher than that of the zeolites, resulting in all of the MOFs with $d_{\text {free }}>10 \AA$ having significantly enhanced linear conversion, compared to the zeolites within the same range. Eleven of the MOF-74 analogues have equal or higher linear conversion than Mg-MOF-274, which is the highest-performing previously synthesized MOF, with the best analogue having a predicted $1.5 \%$ increase in linear conversion over Mg-MOF-274 (see Figure 7). Since there is only one published MOF-74

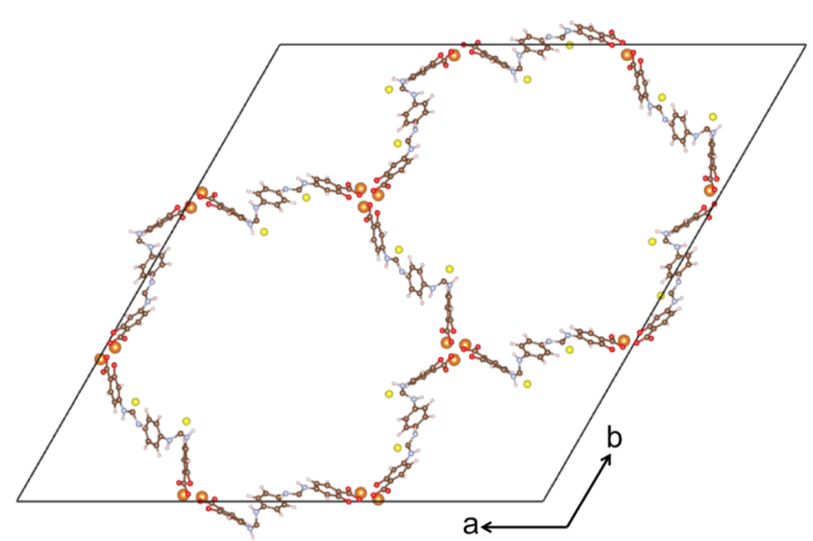

Figure 7. MOF-74 analogue (structure name: $184 \times 1$ all_conf_10850_1, ${ }^{13} d_{\text {free }}=21.0 \AA$ ) with $1.5 \%$ higher linear conversion than Mg-MOF-274, which is the best-performing previously synthesized MOF. analogue structure smaller than $10 \AA\left(\mathrm{Mg}_{2}\right.$ (DHFUMA $\left.)\right),{ }^{36}$ it is not possible to determine the composition-independent effect of pore diameter below $10 \AA$. However, the linear conversion of $\mathrm{Mg}_{2}$ (DHFUMA) falls within the range of the linear conversion among zeolites of the same pore size, suggesting that, for small pore sizes, the physical amount of pore space available to adsorbates dominates their relative free energies of adsorption.

Since the linear conversion is a function of the relative free energies of adsorption of linear and branched isomers, further insights can be gleaned by examining the enthalpic and entropic contributions to these free energies in Figure 8. Both $\Delta H_{\text {ads }}$ and $\Delta S_{\text {ads }}$ for each isomer are dependent very strongly on pore size, with a range of $\Delta H_{\mathrm{ads}}$ and $\Delta S_{\mathrm{ads}}$ values for different isomers inside the same material. $\Delta H_{\text {ads }}$ is very repulsive for small pore sizes, reaches a minimum at $d_{\text {free }} \approx 5 \AA$, the median end-to-end distance of the product isomers, and levels off for larger pore sizes. $\Delta S_{\text {ads }}$ is more monotonic, decreasing sharply for decreasing $d_{\text {free }}<7.5 \AA$, and then remaining relatively constant for larger pore sizes.

While all of the materials appear to have the same average dependence of $\Delta H_{\mathrm{ads}}$ and $\Delta S_{\mathrm{ads}}$ on pore size, the difference between the highest and lowest $\Delta H_{\text {ads }}$ and $\Delta S_{\text {ads }}$ within a single material appears to be slightly larger for the MOFs than for the zeolites, indicating that the MOFs differentiate more between the product isomers than do the zeolites. To investigate this spread, we define the properties $\Delta \Delta H_{\text {ads }}$ and $\Delta \Delta S_{\text {ads }}$, where $\Delta \Delta M_{\mathrm{ads}} \equiv\left\langle\Delta M_{\mathrm{ads}}\right\rangle_{\text {linear }}-\left\langle\Delta M_{\mathrm{ads}}\right\rangle_{\text {branched }}$ for $M=\{S, H\}$. More negative $\Delta \Delta H_{\text {ads }}$ values indicate an enthalpic preference for linear isomers to adsorb in MOFs than in zeolites, while the entropic preference is greater for more positive $\Delta \Delta S_{\text {ads }}$. In Figure $8, \Delta \Delta H_{\text {ads }}$ shows large variations for small pore sizes, indicating that the relative binding energies of linear and branched isomers is very sensitive to pore shape for small pores. For larger pore sizes of $d_{\text {free }}>10 \AA$, the $\Delta \Delta H_{\text {ads }}$ of the MOFs
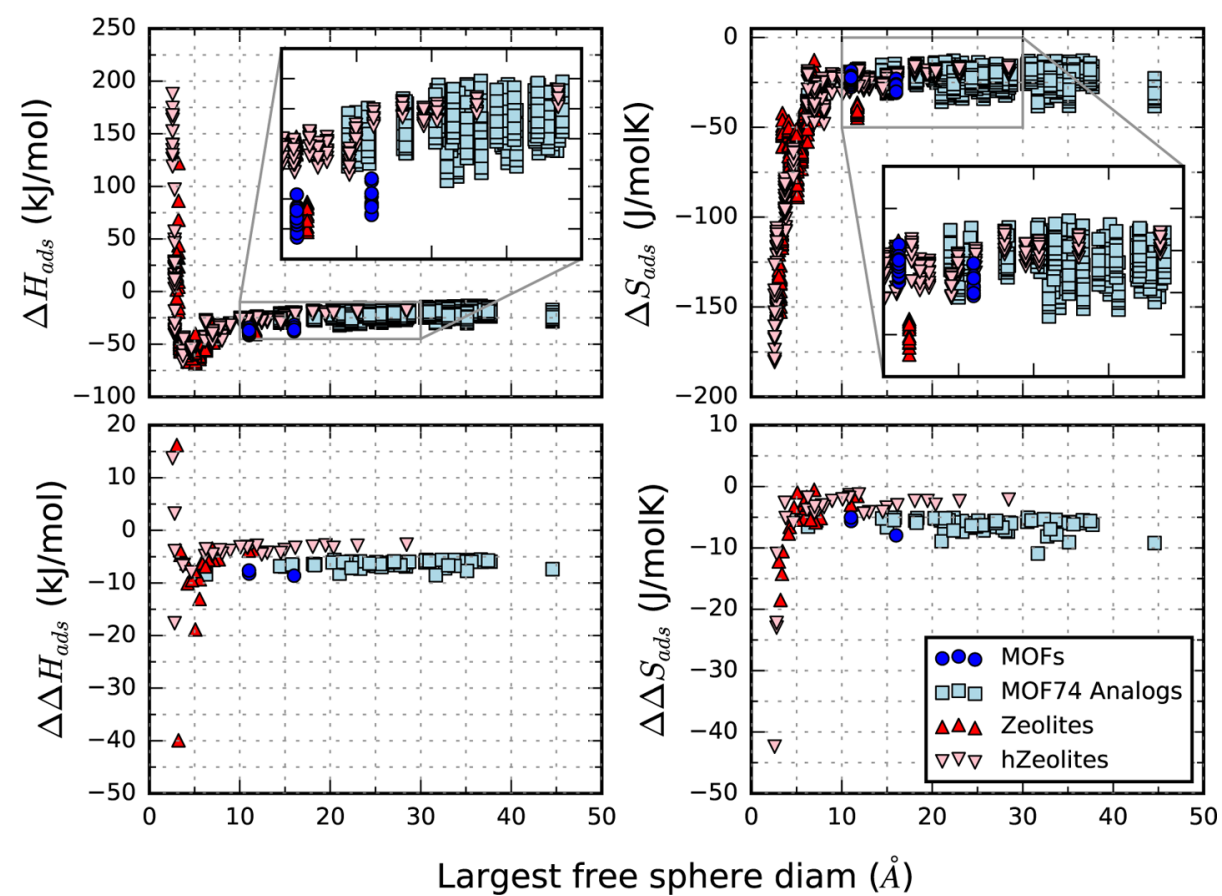

Figure 8. $\Delta H_{\text {ads }}$ and $\Delta S_{\text {ads }}$ of all components (upper); $\Delta \Delta H_{\text {ads }}$ and $\Delta \Delta S_{\text {ads }}$ (lower). $\Delta \Delta M_{\text {ads }} \equiv\left\langle\Delta M_{\text {ads }}\right\rangle_{\text {linear }}-\left\langle\Delta M_{\text {ads }}\right\rangle_{\text {branched }}$ for $M=\{S, H\}$. In the upper two plots, the indicated property is plotted for all 12 product isomers for each of the frameworks considered, with insets for clarity, whereas, in the lower two plots, there is one point per material describing the extent to which each material favors the linear product isomers. 

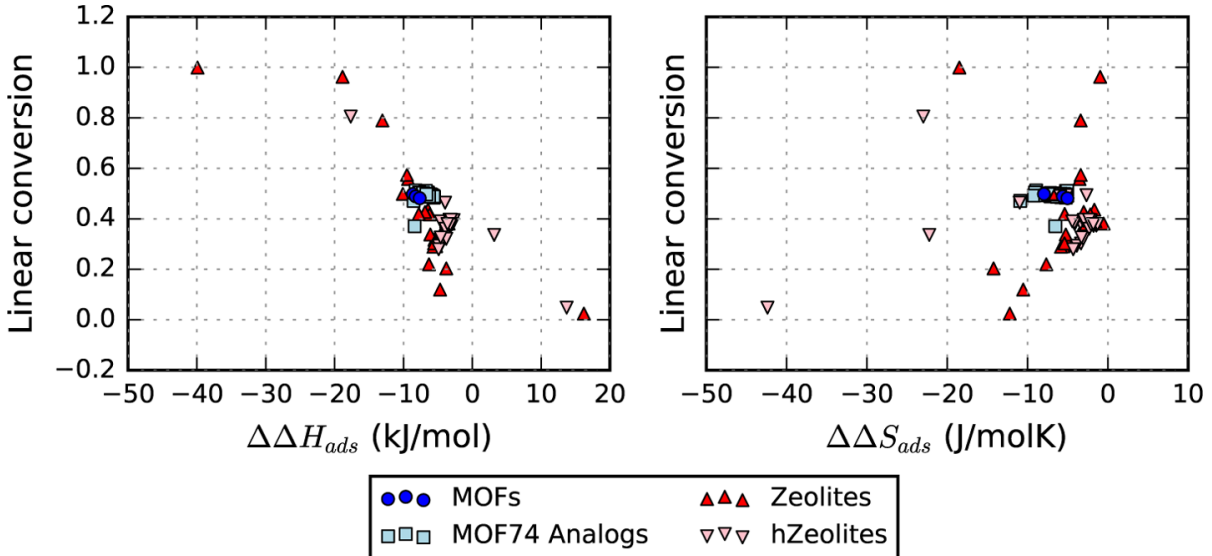

Figure 9. Linear conversion of all frameworks vs $\Delta\left(\Delta H_{\text {ads }}\right)$ (left) and $\Delta\left(\Delta S_{\text {ads }}\right)$ (right). The correlation between linear conversion and $\Delta \Delta H_{\text {ads }}$ of the porous materials indicates that the enthalpy of adsorption is the dominant contributor to differences in linear conversion between materials. By contrast, there is little correlation between linear conversion and $\Delta \Delta S_{\mathrm{ads}}$.

and MOF-74 analogues are consistently $3-5 \mathrm{~kJ} / \mathrm{mol}$ lower than that of zeolite materials, indicating that MOFs enthalpically favor linear isomers. By contrast, from the analogous $\Delta \Delta S_{\text {ads }}$ plot, the MOFs appear to entropically favor the branched isomers. Overall, the $\Delta \Delta H_{\text {ads }}$ value exerts the dominant influence on linear conversion (Figure 9), and the enhanced linear conversion of MOFs can be attributed to enthalpic effects that selectively favor the adsorption of linear isomers.

This suggests that specific chemical interactions can enhance the influence of pore shape on product distribution, adding a layer of complexity to traditional shape selectivity theory, which uses purely geometric considerations to explain differences in product distributions between chemically similar zeolites. ${ }^{11}$ The strong $\pi$-orbital interactions between the double bonds of the olefin isomers and the open metal site in the MOF-74 series enthalpically favors the linear isomers, for which the double bond is more accessible. For the same reason, since linear product isomers have a stronger tendency to adsorb with their $\pi$-bonds facing the open metal site, they lose more configurational entropy upon adsorption, compared to branched isomers.

The effect of these $\pi$-orbital interactions can be seen in the comparison of the binding sites of the double bond in metalorganic and zeolite frameworks (Figure 10). In the Ni-MOF-74, the 1-hexene double bond has a tendency to adsorb closer to open metal sites than the less-accessible double bond in cis-2hexene, which, in turn, is still more localized near the metal sites than cis-3-methyl-2-pentene. In these sites, 1-hexene, cis-2hexene, and cis-3-methyl-2-pentene have $\Delta H_{\text {ads }}$ values of $-38.8,-38.7$, and $-36.5 \mathrm{~kJ} / \mathrm{mol}$, respectively. The same pattern of adsorption site localization, coupled with more favorable $\Delta H_{\text {ads }}$ for the linear isomers, is visible in Mg-MOF-74 and Mg-MOF-274 (see the Supporting Information).

In FAU, since there are no specific framework- $\pi$-bond interactions, there is little difference between the adsorption sites of the three isomers. 1-Hexene, cis-2-hexene, and cis-3methyl-2-pentene have $\Delta H_{\mathrm{ads}}$ values of $-28.8,-30.6$, and $-30.8 \mathrm{~kJ} / \mathrm{mol}$, respectively, opposite to the order of adsorption strength in Ni-MOF-74. As a result, the zeolite disfavors the linear isomers, compared to the MOF. Probability density plots for other frameworks are provided in the Supporting Information. In some of the probability density plots for other zeolites, there is a more pronounced difference between adsorption sites of the branched and linear isomers than in

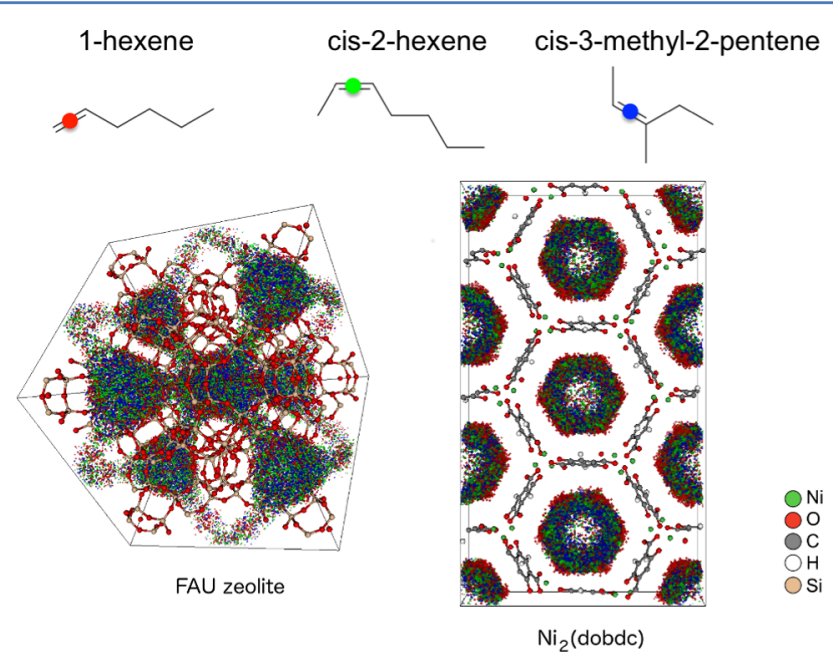

Figure 10. Probability density plots of $\mathrm{C}_{6}$ olefin isomer adsorption sites inside FAU zeolite and Ni-MOF-74. The probability densities correspond to the indicated points marking the geometric center of the $\pi$-bond on the three product isomers shown at the top.

FAU. There are regions with a higher probability of 1-hexene adsorption in AET and hypothetical zeolite h8160847, for example, which are closer to the pore walls and extend into more-constrained pockets in the frameworks. However, they do not coincide with a more favorable $\Delta H_{\text {ads }}$ for linear molecules, compared to the branched ones, as is the case in Ni-MOF-74.

From these observations, we can infer a few design rules to guide the synthesis of new materials for propene dimerization and other olefin oligomerization reactions. To increase the linear conversion of a zeolite material, a smaller framework size of 5-7 $\AA$ can be used. Alternatively, one can also tune the pore chemistry by taking advantage of the large chemical design space of MOFs. A significant enhancement in linear conversion can be achieved by introducing a high density of open metal sites to preferentially bind the double bond of the olefins. $\mathrm{Ni}_{3}$ (BTC) $)_{2}$ also has open metal sites ${ }^{37}$ and could be used as a candidate for this reaction and a template upon which to base analogous structures.

\section{CONCLUSIONS}

In conclusion, we have shown that the product distribution of the propene dimerization reaction in different porous materials 
can be modeled computationally using equilibrium simulations and a three-point charge description of the $\pi$-bond of the olefin double bond. From the free-energy profiles computed for the different materials, we predicted a trend of increased linear conversion in open metal site MOFs, compared to zeolites, which is in good agreement with the linear conversion observed in the experiments. This suggests that the product distribution of the olefin dimerization is largely influenced by the contribution of the pores to the free energy of the product isomers.

We extended our study to other zeolitic and metal-organic frameworks (MOFs), both synthesized and hypothetical, in order to better understand the material properties that influence dimerization product distribution. We found that, for frameworks with a pore diameter under $10 \AA$, selectivity toward linear isomers is a sensitive function of pore size, with very high linear conversion at $\sim 5 \AA$. In this regime, the effect of pore size on the product distribution can be attributed to changes in the enthalpy of adsorption. It is important to note that, in industrial applications, a framework with such a small pore size might be disadvantageous, because of the diffusive barriers that would result.

Above a pore diameter of $10 \AA$, the linear conversion for the zeolite materials plateaus at $\sim 40 \%$, while the linear conversion for the MOFs increases and remains steady at $\sim 50 \%$, with a few frameworks even predicted to have higher linear selectivity than the experimentally high-performing Ni-MOF-74. This striking enhancement in linear conversion for MOFs is due to the preferential adsorption of $\pi$-bonds at open metal sites.

These results elucidate some of the mechanisms governing product distribution in the propene dimerization reaction, and suggest promising not-yet-synthesized frameworks that could deliver higher linear conversion than the existing catalysts. Further work is needed to investigate the product distribution over different classes of open metal sites (for example, copper paddlewheel-type structures) to determine whether the linear enhancement effect is specific to the open metal site motif in the MOF-74 family. In addition, in this study, we only took into account thermodynamic considerations in predicting the linear conversion in different materials. For practical applications, there are many other factors, such as framework stability and diffusion limitations, which we did not address. Additional theoretical and experimental studies could clarify the influence of these phenomena on the product distribution.

\section{ASSOCIATED CONTENT}

\section{S Supporting Information}

The Supporting Information is available free of charge on the ACS Publications website at DOI: 10.1021/acscatal.7b00712.

Force field information, adsorbate probability density plots, and material descriptor data; download and visualization of screening data available at: http://www. materialscloud.org/archive/2017.0004/v1/ (PDF)

\section{AUTHOR INFORMATION}

\section{Corresponding Author}

*E-mail: berend.smit@epfl.ch.

\section{ORCID}

Yifei Michelle Liu: 0000-0002-4705-6664

Berend Smit: 0000-0003-4653-8562

Notes

The authors declare no competing financial interest.

\section{ACKNOWLEDGMENTS}

This project has received funding from the European Research Council (ERC), under the European Union's Horizon 2020 research and innovation program (Grant No. 666983, MaGic). Y.M.L. acknowledges support from an NSF Graduate Research Fellowship.

\section{REFERENCES}

(1) Furukawa, H.; Cordova, K. E.; O’Keeffe, M.; Yaghi, O. M. Science 2013, 341, 1230444.

(2) Alaerts, L.; Séguin, E.; Poelman, H.; Thibault-Starzyk, F.; Jacobs, P. A.; De Vos, D. E. Chem.-Eur. J. 2006, 12, 7353-7363.

(3) Lee, J.; Farha, O. K.; Roberts, J.; Scheidt, K. A.; Nguyen, S. T.; Hupp, J. T. Chem. Soc. Rev. 2009, 38, 1450-1459.

(4) Dang, G. H.; Lam, H. Q.; Nguyen, A. T.; Le, D. T.; Truong, T.; Phan, N. T. S. J. Catal. 2016, 337, 167-176.

(5) Liu, J.; Chen, L.; Cui, H.; Zhang, J.; Zhang, L.; Su, C.-Y. Chem. Soc. Rev. 2014, 43, 6011-6061.

(6) O’Connor, C. T.; Kojima, M. Catal. Today 1990, 6, 329-349.

(7) Wilshier, K.; Smart, P.; Western, R.; Mole, T.; Behrsing, T. Appl. Catal. 1987, 31, 339-359.

(8) Quann, R. J.; Green, L. A.; Tabak, S. A.; Krambeck, F. J. Ind. Eng. Chem. Res. 1988, 27, 565-570.

(9) Mlinar, A. N.; Ho, O. C.; Bong, G. G.; Bell, A. T. ChemCatChem 2013, 5, 3139-3147.

(10) Mlinar, A. N.; Keitz, B. K.; Gygi, D.; Bloch, E. D.; Long, J. R.; Bell, A. T. ACS Catal. 2014, 4, 717-721.

(11) Smit, B.; Maesen, T. L. M. Nature 2008, 451, 671-678.

(12) Smit, B.; Maesen, T. L. M. Chem. Rev. 2008, 108, 4125-4184.

(13) Witman, M.; Ling, S.; Anderson, S.; Tong, L.; Stylianou, K. C.; Slater, B.; Smit, B.; Haranczyk, M. Chem. Sci. 2016, 7, 6263-6272.

(14) Baerlocher, C.; McCusker, L. B. Database of Zeolite Structures; 2007. Available via the Internet at: http://www.iza-structure.org/ databases/.

(15) Earl, D. J.; Deem, M. W. Ind. Eng. Chem. Res. 2006, 45, 54495454.

(16) Böhme, U.; Barth, B.; Paula, C.; Kuhnt, A.; Schwieger, W.; Mundstock, A.; Caro, J.; Hartmann, M. Langmuir 2013, 29, 85928600.

(17) Wick, C. D.; Martin, M. G.; Siepmann, J. I. J. Phys. Chem. B 2000, 104, 8008-8016.

(18) Mayo, S. L.; Olafson, B. D.; Goddard, W. A., III. J. Phys. Chem. 1990, 94, 8897-8909.

(19) Rappé, A. K.; Casewit, C. J.; Colwell, K. S.; Goddard, W. A., III; Skiff, W. M. J. Am. Chem. Soc. 1992, 114, 10024-10035.

(20) Krishna, R.; van Baten, J. M. Phys. Chem. Chem. Phys. 2011, 13, 10593.

(21) Lamia, N.; Jorge, M.; Granato, M. A.; Almeida Paz, F. A.; Chevreau, H.; Rodrigues, A. E. Chem. Eng. Sci. 2009, 64, 3246-3259.

(22) Yazaydın, A. O.; Snurr, R. Q.; Park, T.-H.; Koh, K.; Liu, J.; LeVan, M. D.; Benin, A. I.; Jakubczak, P.; Lanuza, M.; Galloway, D. B.; Low, J. J.; Willis, R. R. J. Am. Chem. Soc. 2009, 131, 18198-18199.

(23) Campaná, C.; Mussard, B.; Woo, T. K. J. Chem. Theory Comput. 2009, 5, 2866-2878.

(24) Mercado, R.; Vlaisavljevich, B.; Lin, L.-C.; Lee, K.; Lee, Y.; Mason, J. A.; Xiao, D. J.; Gonzalez, M. I.; Kapelewski, M. T.; Neaton, J. B.; Smit, B. J. Phys. Chem. C 2016, 120, 12590-12604.

(25) Liu, B.; Smit, B.; Rey, F.; Valencia, S.; Calero, S. J. Phys. Chem. C 2008, 112, 2492-2498.

(26) Weitz, S. L.; Potoff, J. J. Fluid Phase Equilib. 2005, 234, 144150.

(27) Wang, S.; Yang, Q.; Zhong, C. Sep. Purif. Technol. 2008, 60, 3035.

(28) Evans, M. G.; Polanyi, M. Trans. Faraday Soc. 1936, 32, 13331360.

(29) Frenkel, D.; Smit, B. Understanding Molecular Simulation: From Algorithms to Applications, 2nd Edition; Academic Press: San Diego, CA, 2001; pp 280-282, 331-336. 
(30) Smit, B.; Siepmann, J. I. J. Phys. Chem. 1994, 98, 8442-8452.

(31) Vlugt, T. J. H.; García-Pérez, E.; Dubbeldam, D.; Ban, S.; Calero, S. J. Chem. Theory Comput. 2008, 4, 1107-1118.

(32) Dubbeldam, D.; Calero, S.; Ellis, D. E.; Snurr, R. Q. Mol. Simul. 2016, 42, 81-101.

(33) Childs, H.; Brugger, E.; Whitlock, B.; Meredith, J.; Ahern, S.; Pugmire, D.; Biagas, K.; Miller, M.; Harrison, C.; Weber, G. High Performance Visualization: Enabling Extreme-Scale Scientific Insight; CRC Press: Boca Raton, FL, 2012; pp 357-372.

(34) Rosi, N. L.; Kim, J.; Eddaoudi, M.; Chen, B.; O’Keeffe, M.; Yaghi, O. M. J. Am. Chem. Soc. 2005, 127, 1504-1518.

(35) McDonald, T. M.; Lee, W. R.; Mason, J. A.; Wiers, B. M.; Hong, C. S.; Long, J. R. J. Am. Chem. Soc. 2012, 134, 7056-7065.

(36) Witman, M.; Ling, S.; Gladysiak, A.; Stylianou, K. C.; Smit, B.; Slater, B.; Haranczyk, M. J. Phys. Chem. C 2017, 121, 1171-1181.

(37) Yaghi, O. M.; Li, H.; Groy, T. L. J. Am. Chem. Soc. 1996, 118, 9096-9101. 